\title{
Editorial
}

\section{Tuberculosis \& Diabetes: need to be addressed}

Over the past two decades, TB control programmes worldwide including SAARC region have implemented TB control through DOTS and the Stop TB Strategy with evident success, including substantial increase in rates of case detection and improved treatment outcomes.

To achieve more outcome, innovative plan, policy and implementation at grass root level have to be lunched keeping in mind of best practices, at community level. Similarly, programme has to reach to un-reached population with easy availability of resources at community level so that hidden and unidentified cases can get easy service delivery. In addition, NTP has to address TB not only as a separate disease but its association and impact of other diseases like diabetes \& others on it.

Tuberculosis and diabetes mellitus are two major public health problems, which not only often coexist but have serious implication on each other. Diabetes mellitus has an impact on symptomatology, radiological presentation, diagnosis and management of TB.

Tuberculosis (TB) kills more than 3,500 people each day worldwide, leading to approximately 1.4 million deaths every year. One-third of the world's population is currently infected with the causative agent of TB, and 8.8 million new cases of active TB are estimated to occur around the world each year. TB is fueled by several social and economic factors, such as poverty or malnutrition, as well as other infectious diseases, such as HIV.

Diabetes patients are three times more likely to develop of TB when infected. In India alone, $15 \%$ of TB is attributed to diabetes. Consequently, rates of TB are higher in people with diabetes than in the general population, and diabetes is a common morbidity in people with TB. Diabetes can worsen the clinical course of TB and can worsen glycogenic control in people with diabetes. Individuals with both conditions thus require careful clinical management. Strategies are needed to ensure that optimal care is provided to patients with both diseases: TB must be diagnosed early in people with diabetes, and diabetes must be diagnosed early in people with TB. Changes in lifestyle and diet have contributed to an increased prevalence of diabetes in many low-income and middle-income including SAARC countries where the burden of TB is high. The growing burden of diabetes is contributing to sustained high levels of TB in the community, and the proportion of TB cases attributable to diabetes globally is likely to increase over time. This double burden of disease is a serious and growing challenge for health systems.

In recent years, strong evidence has been gathered to confirm a link between TB and yet another disease: diabetes mellitus. That link had been suspected for centuries. Many studies now show that diabetes may be associated with an increased risk of developing active TB, and that TB patients who also have uncontrolled diabetes may have higher rates of treatment failure and death.

The World Health Organization, the International; Union Against TB and Lung Diseases (the Union), in collaboration with other partners, national TB control programmes developed the Collaborative Framework for Care and Control of TB and Diabetes based on the systematic reviews on the link between TB and Diabetes. Thus, both need to be managed properly at the earliest possible in order to achieve favorable treatment out comes of tuberculosis by carrying surveillance of TB disease prevalence among people with diabetes and by Intensifying detection of TB among people with diabetes.

TB control programs should also develop educational materials to be distributed at diabetes treatment centers, to inform diabetic patients of their risk of developing active TB. More specifically, symptoms of TB should be clearly highlighted to advise diabetic patients when to seek TB screening. More over the millennium Development goal specifies that the incidence of infectious diseases such as TB should be halted and reversed by 2015. To succeed in achieving this target, it is important to focus on resource-poor countries not only on for HIVIAIDS but also on diabetes as a significant epidemiological risk factor. 